\title{
Photoinduced SHG in Push-Pull Chromophores 1,3-dithiol-2-yliedene Moieties Embedded in Photopolymer Matrices
}

\author{
A. Migalska-Zalas, M. Makowska-Janusik, I.V. Kityk \\ Institute of Physics, al. Armii Krajowej 13/15, 42-201 Częstochowa, Poland
}

B. SAHRAOUI

Laboratory POMA, UMR CNR 6136, University of Angers, France

M. Salle ANd A. Gorgues

Laboratory IMMO, UMR CNRS 6501, University of Angers, France

\begin{abstract}
Photoinduced optical second harmonic generation was demonstrated in the several dithiole chromophore molecules with different degree of acceptor function. Theoretical quantum chemical simulations and experimental photoinduced second harmonic generation measurements showed that additional aromatic rings within the dithiole chromophore molecule play an important role in the pump-power dependencies of the second harmonic generation. Varying the presence of the aromatic rings one can achieve substantially different photoinduced second-order nonlinear optical properties.
\end{abstract}

PACS numbers: 42.65.An, 42.65.Ky

\section{Introduction}

One can recently observe an increasing interest in organic materials used in nonlinear optics (NLO) [1,2]. Most of investigations are devoted to the second-order NLO effects, particularly to optical second harmonic generation (SHG). The SHG needs presence of noncentrosymetric charge density distribution. The traditional way to obtain the required noncentrosymetric material is to incorporate the organic molecule (guest chromophore) into the polymer matrices (host) [3]. Using a 
guest-host technique one can obtain an alignment of the guest material. Additional molecular poling can be obtained by photoinducing process, too. The photopoling of the disordered materials (glasses, polymers, cements etc.) can found wide applications in optics or optoelectronics.

The present work is devoted to investigations of additional aromatic rings on the photoinduced optical SHG (PISHG) of 1,3-dithiol-2-ylidene incorporated within the oligoetheracrylate matrix. The main goals of the present work are the following:

- to investigate the influence of inter- and intramolecular interaction in the 1,3-dithiol-2-ylidene with and without the aromatic rings on the local noncentrosymmetry in the space charge density distribution experimentally (using $\mathrm{SHG}$ ) as well as theoretically (by quantum chemical calculations),

- to simulate the photoinduced SHG behaviour depending on the photoinducing power.

\section{Experimental and calculation methods}

\subsection{Experimental methods}

The synthesized chromophores samples had a powder-like form with diameter of $10-20 \mu \mathrm{m}$. Synthesis of the molecule was performed by a method described in Ref. [4]. They were a solute in the oligoetheracrylate photopolymer matrix. More technical details are presented in Ref. [3]. The solidification process was performed using a nitrogen laser with a power about $50 \mathrm{~W} / \mathrm{cm}^{2}$ and electric field was used to align the studied compounds (see Fig. 1) in order to enhance their dipole moments. The degree of nonhomogeneity of chromophore distribution was equal to about $0.24 \%$.

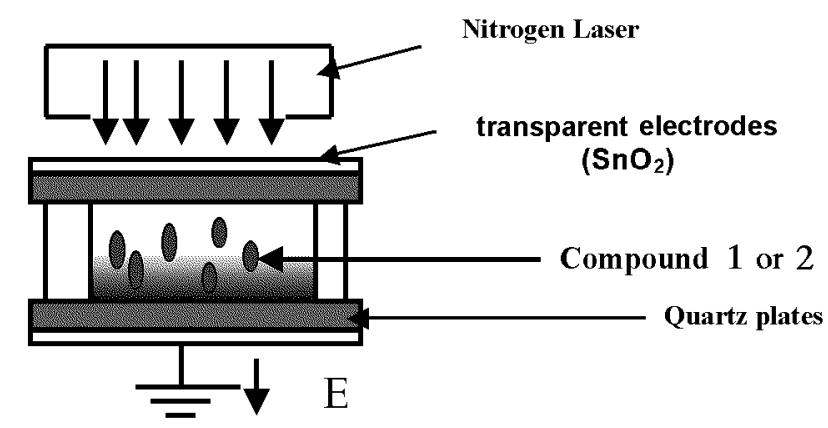

Fig. 1. The process of photopolymerization was carried out under applied electric field ( $E$ was varied from 300 to $800 \mathrm{kV}$ ). Solidification of the liquid composition was obtained using UV-light generated by nitrogen laser for about $3 \mathrm{~min}(\lambda=337 \mathrm{~nm}$ and the used power was about $\left.35 \mathrm{~W} / \mathrm{cm}^{2}\right)$. 
For the performing of photoinduced measurements of nonlinear optical susceptibilities we used experimental setup described in Ref. [5] and its principal scheme is presented in Fig. 2. The photoinduced changes (optical pumping) was achieved using the UV nitrogen laser $(\lambda=337 \mathrm{~nm}, \tau=0.50 \mathrm{ps}, P=70-90 \mathrm{MW}$ ). An apparatus for SHG detection was supplied with a focused beam from a single-mode picosecond $\mathrm{Nd}$ :YAG laser (probe laser), with power of $30 \mathrm{MW}$, a pulse time duration of $10-25 \mathrm{ps}$, with fundamental wavelength $1.06 \mu \mathrm{m}$. The measurements were performed for polarized light beam.

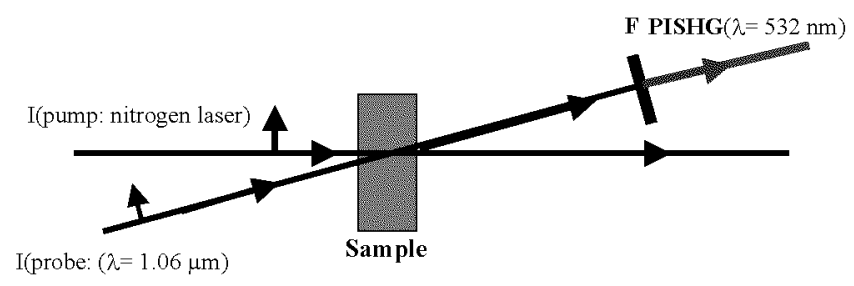

Fig. 2. The principal scheme of experimental setup used for the measurements of PISHG. We use $Q$-switched nitrogen laser as a pump beam ( $\lambda=337 \mathrm{~nm}, t=5-45 \mathrm{ps}$; power was varied up to the $\left.1.2 \mathrm{GW} / \mathrm{cm}^{2}\right)$ and $\mathrm{Nd}$ :YAG laser as probe beam $(\lambda=$ $1.06 \mu \mathrm{m} ; t=30 \mathrm{ps}$, power of probe beam $30 \mathrm{MW}$ ). Filter $\mathrm{F}$ is used in order to cut $\lambda=1.06 \mu \mathrm{m}$. The measurements were performed for polarized light beam.

The proposed photoinduced SHG has substantial advantages comparing with the traditional electric-field induced $\mathrm{SHG}$ because we can apply external electric field even for the conducting composites, which in our case are crucial. At the same time this method allows to perform the measurements in the dynamic pump-probe regime which is impossible in the case of the electric-induced SHG effects.

\subsection{Theoretical simulations}

For theoretical simulations of photoinduced SHG two different chromophore molecules (molecule indicated by $\mathbf{1}$ - Fig. 3a and by $\mathbf{2}-$ Fig. 3b) of 1,3-dithiol-2-yliedene were used. Initially geometry optimization (searching of the total energy minimum) of molecule was performed. The $\mathrm{MM}^{+}$molecular force field method was used for total energy minimization $[6,7]$. The performed simulations have shown that one-plane molecular fragments are more preferable. All quantum chemical calculations were performed by semi-empirical ZINDO/S method in the restricted Hartree-Fock option and convergence limit up to $10^{-6} \mathrm{eV}$ after 500 iterations was achieved.

The considered model is assumed on the relatively high intramolecular space localization of the observed photoinduced changes. Local perturbations are considered only within a framework of the separated (isolated) molecule. Therefore 
(a)

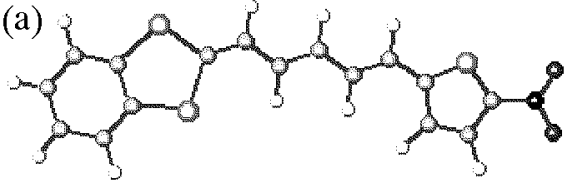

(b)

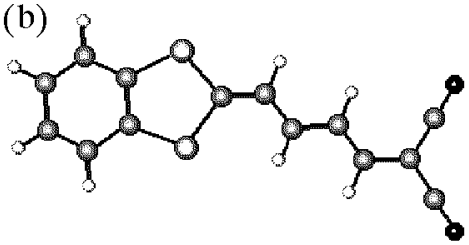

Fig. 3. Chemical formula of the investigated molecules: molecule number 1 (a), molecule number 2 (b).

deviations from the experimental data will give information concerning the observed intramolecular and intermolecular interactions. Influence of the intermolecular electron vibration as well long-range solid state interactions is not taken into account.

\section{Results and discussion}

We measured the photoinduced SHG intensities as a function of nitrogen pump laser power density for two different kinds of samples. From Fig. 4a one can easily observe that for the sample 1 the SHG output signal increases as function of power of the photoinducing nitrogen laser pulses and achieves its maximum at the pumping power density for about $1.8 \mathrm{GW} / \mathrm{cm}^{2}$. For the sample 2 we can observe similar behaviour of SHG output (Fig. 4b). The experimental $\beta_{x x x}$ was deduced from the measurement of second-order susceptibilities.
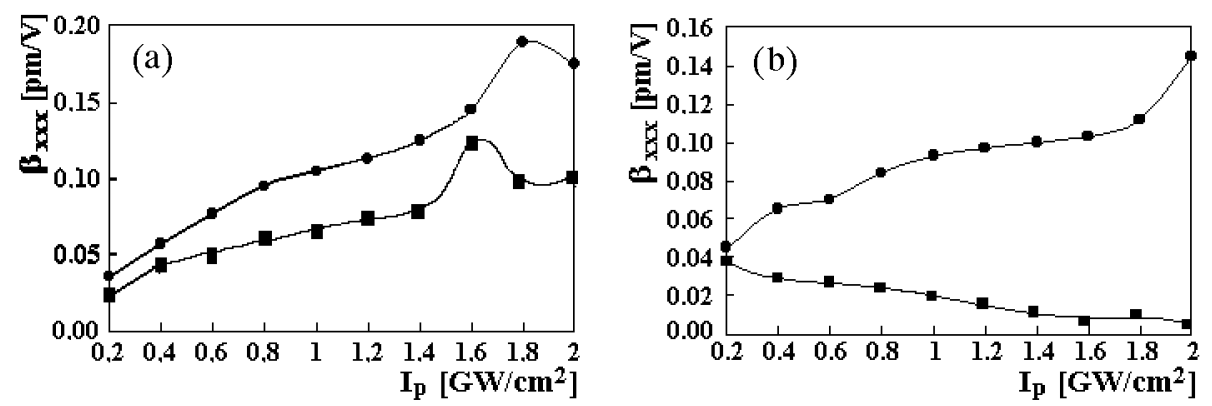

Fig. 4. The photoinduced PISHG output signal for first (a) and second sample (b), experimental results - line with circles, experimental data for the intramolecular hyperpolarizabilities in arbitrary units - line with squares (theoretical results), versus the power of photoinduced photon flux.

For the samples denoted as $\mathbf{2}$, from Fig. 4b we can observe that theoretical curve is not so sharp like experimental one. The obtained experimental data for compound 1 show good agreement with theory. It is necessary to emphasize a good correlation of the photoinduced behaviour of the hyperpolarizability $\beta_{x x x}$ 
obtained from the theoretical simulations and measured experimentally. The used theoretical formula of the hyperpolarizability $\beta_{x x x}$ is expressed as follows:

$$
\beta_{i j k}(0 \rightarrow 1) \cong \frac{\mu^{2} \Delta \mu}{\left(E_{\text {HOMO }}-E\right)_{j}^{4}},
$$

where $\mu$ are the transition dipole moments between the high occupied molecular orbital (HOMO) $E_{\text {HOMO }}$ and the first excited state $E ; \Delta \mu$ - difference of the state dipole moments between the excited configuration interaction (CI) level and the HOMO energy level. Indices $i, j$, and $k$ are defined as laboratory coordinate setup, particularly $x$-axis corresponds to the longest axes of the molecule, $y$-axis corresponds to the axes perpendicular to the $y$ within the molecular plane of the molecule.

We can expect that for the first sample the electronic intramolecular subsystem plays a key role in the observed photoinduced changes.

From Table one can see that one additional aromatic ring (specimen 1) decreases HOMO-LUMO (lowest occupied molecular orbital) energy splitting gap comparing to sample number $\mathbf{2}$. For both samples HOMO-LUMO energy splitting gaps decrease with the increase in the photon power density. However, behaviour of the LUMO of the first sample is completely different than the behaviour of the second sample (see Fig. 5). The presence of additional aromatic ring decreases transparency of the sample. The spectral difference between positions of first absorption peaks for both samples is about $100 \mathrm{~nm}$.

TABLE

Changes of the HOMO-LUMO energy splitting gap for samples number 1 and $\mathbf{2}$ versus the power of pumping beam.

\begin{tabular}{c|c|c}
\hline \hline \multirow{2}{*}{$\begin{array}{c}I_{p} \\
{\left[\mathrm{GW} / \mathrm{cm}^{2}\right]}\end{array}$} & \multicolumn{2}{|c}{ HOMO-LUMO gap [eV] } \\
\cline { 2 - 3 } & Sample 1 & Sample 2 \\
\hline 0 & 5.438932 & 6.249419 \\
0.2 & 5.354674 & 6.225095 \\
0.4 & 5.353045 & 6.224140 \\
0.6 & 5.351424 & 6.223403 \\
0.8 & 5.350228 & 6.222777 \\
1 & 5.349171 & 6.222225 \\
1.2 & 5.348211 & 6.221722 \\
1.4 & 5.347329 & 6.221260 \\
1.6 & 5.346506 & 6.220828 \\
1.8 & 5.345730 & 6.220421 \\
2 & 5.344996 & 6.220035
\end{tabular}




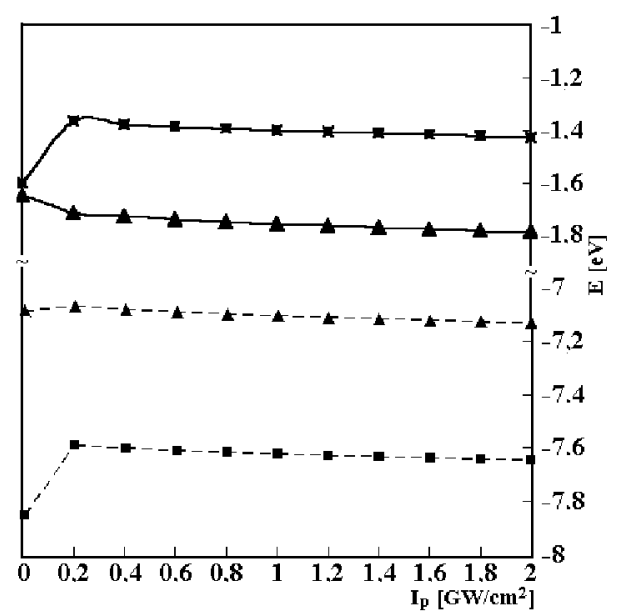

Fig. 5. Changes of the HOMO (dashed lines) and LUMO (solid lines) energy values for the sample $\mathbf{1}$ - lines with triangles and for the sample $\mathbf{2}$ - lines with squares versus the photoinduced photon power.

The SHG output signal depends on the difference between ground and excited dipole moment (see Eq. (1)). The ground dipole moment of the sample $\mathbf{1}$ is lower than the one of sample $\mathbf{2}$, but the dipole moment for the first excited state is higher for the sample $\mathbf{1}$ than for the second one. Therefore the difference between dipole momentum for the molecule $\mathbf{1}$ is higher than for the $\mathbf{2}$ and as a consequence the SHG output signal is higher for the sample $\mathbf{1}$ than for the $\mathbf{2}$. We can see a similar behaviour from the electron distributions in ground and excited states for both molecules (Fig. 6). The asymmetry in the electron distribution for ground and excited states plays key role in the observed nonlinear optical susceptibilities.

For the molecule $\mathbf{1}$ the electronic contribution plays dominant role in the observed phenomenon. For the molecule 2 pump-dependent behaviour of the photoinduced SHG output signal is completely different for theoretical calculations than for experimental work. In proposed theoretical simulation only electronic system was taken into account. From Fig. 3 b one can conclude that for the molecule 2 the vibration and intermolecular contributions can be very important. The right side of molecule ( $\mathrm{N}-\mathrm{O}$ doubled and tripled bonds and the additional aromatic ring) plays important role in the vibration system.

The second reason of the disagreement between the theoretical and experimental results of SHG signal output for second molecule could be quantum chemical calculations without interaction between molecule and polymer matrix. For the same kind of materials the host-guest interface substantially affects dipole momentum. For isolated molecule number $\mathbf{2}$ transition dipole momentum decreases with the increase in the powder of photoinduced photon flux. Moreover, it may lead to changes of effective charges of the molecule [8]. 

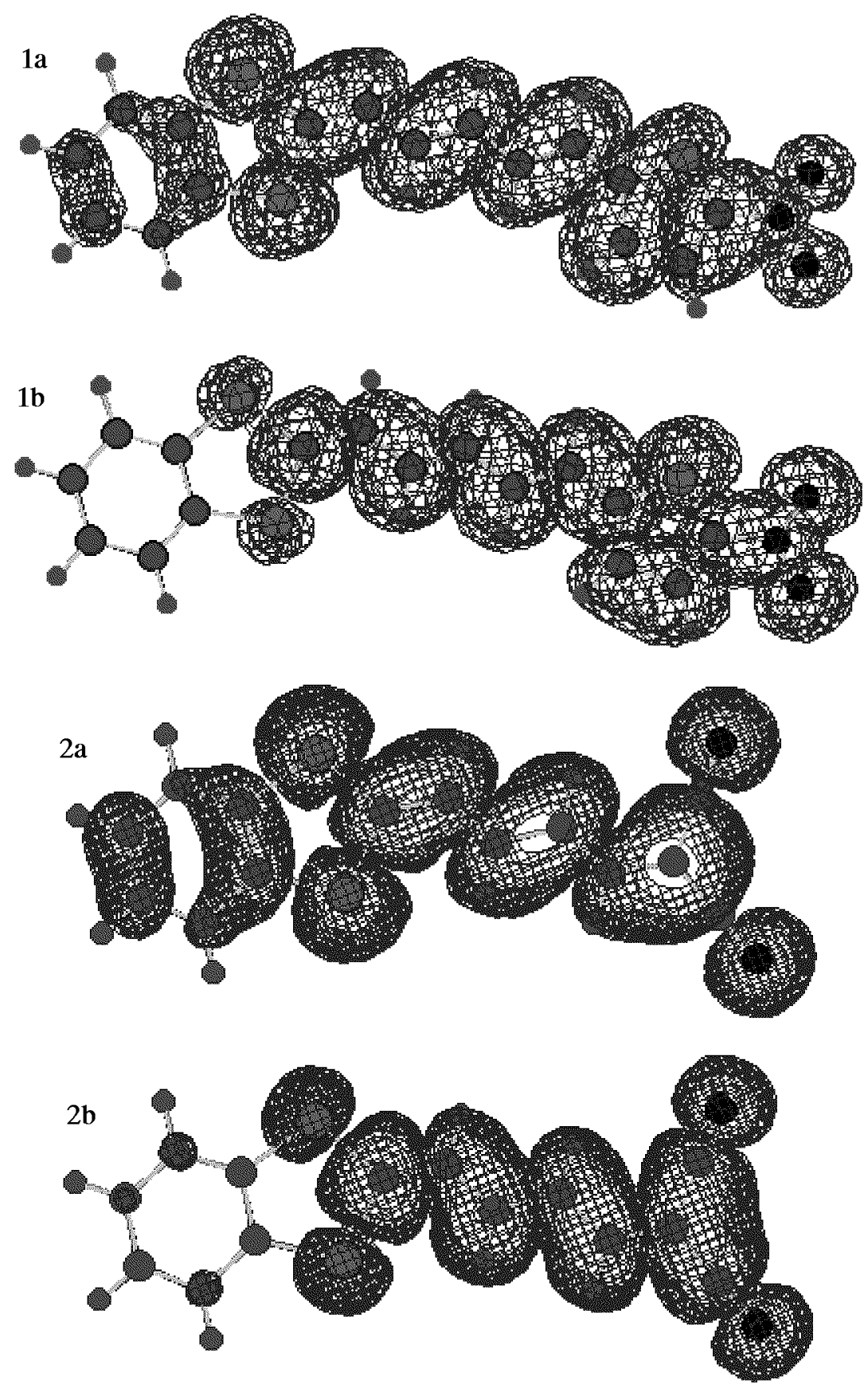

Fig. 6. Charge density distribution of the electrons for two different chromophores: for ground state of the $\mathbf{1}$ and $\mathbf{2}$ sample (1a and $\mathbf{2 a}$, respectively) and first excited state (1b and $\mathbf{2 b}$, respectively). 
The investigations of the observed discrepancies will be a subject of separate work.

\section{Conclusions}

Theoretical quantum chemical simulations and experimental photoinduced SHG measurements have shown that additional aromatic rings within the dithiole chromophore molecule play an important role in the pump-power dependencies of the SHG. Varying the presence of the aromatic rings one can achieve substantially different photoinduced second-order nonlinear optical properties.

\section{References}

[1] P.N. Prasad, D.J. Wiliams, Introduction to Nonlinear Optical Effects in Molecules and Polymers, Wiley, New York 1990; E.J. Baerends, G. Ricciardi, A. Rossa, Coord. Chem. Rev. 230, 5 (2002).

[2] Ch. Bosshard, K. Sutter, Ph. Pretre, J. Hulliger, M. Florsheimer, P. Kaatz, P. Gunter, Organic Nonlinear Optical Materials, Vol. 1, Gordon and Breach, Amsterdam 1995.

[3] R.J. Mervinskii, I.V. Kityk, M. Makowska-Janusik, J. Straube, J. Nonlinear Opt. 13, 245 (1995).

[4] T.T. Nguyen, M. Salle, J. Delaunay, A. Riou, P. Richomme, J.M. Raimundo, A. Gorgues, I. Ledoux, C. Dhenaut, J. Zyss, J. Ordun, J. Garin, J. Mater. Chem. 8, 1185 (1998).

[5] J. Wasylak, J. Kucharski, E. Golis, M. Makowska-Janusik, A. Mefleh, I.V. Kityk, Opt. Mater. 12, 429 (1999).

[6] S.J. Weiner, P.A. Kollman, D.A. Case, U.C. Ghio, G. Alagona, J.S. Profeta, P. Weiner, J. Am. Chem. Soc. 106, 765 (1984).

[7] S.J. Weiner, P.A. Kollman, D.T. Nguyen, D.A. Case, J. Comput. Chem. 7, 230 (1986).

[8] I.V. Kityk, B. Sahraoui, I. Ledoux-Rak, M. Salle, A. Migalska-Zalas, T. Kazuo, A. Gorgues, Mater. Sci. Eng. B 87, 148 (2001). 